\title{
Predicting Theropod Hunting Tactics Using Machine Learning
}

\author{
Matthew Charles Millar ${ }^{1 *}$ \\ ${ }^{1}$ Northcentral University, Arizona, USA \\ *Corresponding author: Matther Millar: matthew.c.r.millar@gmail.com
}

\section{OPEN ACCESS}

Citation: Millar M.C. (2019)

Predicting Theropod Hunting

Tactics Using Machine Learning.

Open Science Journal 4(1)

Received: $10^{\text {th }}$ September 2018

Accepted: 20 ${ }^{\text {th }}$ September 2019

Published: $7^{\text {th }}$ September 2019

Copyright: (c) 2019 This is an open access article under the terms of the Creative Commons Attribution

License, which permits unrestricted use, distribution, and reproduction in any medium, provided the original author and source are credited.

Funding: The author(s) received no specific funding for this work

Competing Interests: The author have declared that no competing interests exists.

\section{Abstract:}

The use of machine learning in different fields is becoming a more common practice thanks to Big Data and better granularity in data collection. The application of machine learning to animal behavioral pattern analysis is becoming more popular due to the increase in size, types, and quality of data. Machine learning can even be used to predict the actual behavior of animals based off certain features. This approach can also be useful for predicting the behavior of extinct animals. This paper is the goal is to explore the possibility of using machine learning techniques to predict the hunting habits of dinosaurs based solely off physical characteristic of the animal. By using the biomechanical features, a model can be created to aid in the classification of animals into either a scavenger or hunter roles. The results from the test show that there is a strong correlation between the physical characteristics and potential hunting habits. The models used here can then be a good baseline in predicting other theropods based solely on their body's physical characteristics. The T-Rex was used as the test subject and was correctly classified as a primary hunter in most of the models.

Keywords: Machine Learning, Artificial intelligence, Animal behavior modeling, Tyrannosaurus Rex, Hunting behavior modeling 


\section{Introduction}

Dinosaurs have captured the imaginations and interest of everyone at some point in time. The largest animals that have ever roamed the earth still have many mysteries the surround them, especially when it comes to behavior patterns. For paleontologist, life, eating habits, family lives, and hunting methods come from looking at fossils which are the primary source of records of dinosaur's behavior patterns. By basing these behaviors on the pose of the fossils were found in, the habitat that they lived in, the animals bio-mechanics, and mainly with comparison with modern-day animals that are either similar in physical characteristics or share similar ecological functions. This forces paleontologist to use speculation, which increases the disagreements between experts in the field. The majority of thinking in the paleontology society is that dinosaur's behavior would most likely follow or be very similar to their closest living relatives, birds, and crocodiles. Biomechanics has provided the best level of insight into how dinosaur lived and behaved based on their skeletal structure and how they were able to move, run, and interact with each other [1], [2], [3].

Machine learning (ML) algorithms have only recently seen use in animal behavior pattern mapping. ML models can prove to be an excellent method for the prediction and categorization of animal's behavior based off physical characteristics or biomechanics, which can control the animal's behavior to a degree. By taking these algorithms and applying them to dinosaurs, this study hopes to define a new tool and architecture for the use of paleontologist to help aid in the classification of each dinosaur's behavior patterns base samples from their fossils and biomechanics.

\section{Problem Statement}

The controversy of if a dinosaur was either a primary hunter or scavenger has gone on for years. There is seldom consensus on if a dinosaur can survive on just eating pure carrion or if they hunt actively for prey [4]. There have been many cases where it is challenging to determine to what extent an animal relies on hunting over scavenging as there are very few actual cases of in the wild of pure obligatory hunters or scavengers. Both hunters and scavengers share similar traits, but some traits are more pronounced in hunters over scavengers as these traits are designed to kill over simple searching [5].

Goal:

The purpose of this study is a pilot study to showcase the use of ML algorithms to aid in the classification of dinosaur hunting behavior to determine the extent of hunting over scavenging for gathering resources.

Background:

Charles Darwin, John Way, and Charles LeRoy are the fathers of evolution and animal behavior studies. These men believed that only through extensive observation over a long period in the animal's natural habitat can the complexity of animal behavior be studied. The study of animals broke into two different methods, ecology and ethology. Ethology looks at the biological reasons behind the animal's behavioral patterns. Ecology looks at how the animal interacts with its environment [6].

Ethology is the science that studies animal behavior in an objectively. The focus of ethology is why certain behaviors offer an evolutionarily beneficial trait. 
Behaviourism is another method for studying animal behavior with the focus on scientifically measured responses to certain stimuli or other trained responses. These two methods varied in one look at how behavior or trait affects a species evolution either benefiting or not, while the other method looks at how to species reacts to a given situation. An animal behaviorist may also look at instincts of a species of animal which is an inherited response to a specific stimulation without any reason or training [7], [8], [9].

Methods of collecting data and analysis in ethological studies originally were as simple as a pencil and paper to write notes and to help in quantifying behaviors in animals, but this has now given way to more accurate and more accessible to use methods of using computer and device assistance to create large amounts of data. Statistics have also played a key role in quantifying animal behavior. Such methods as frequency, latency, duration, and even the number of different types of behaviors can be measured [10]. These methods tend to stray away from computer simulations and focus on pure statistical models and physical counts rather than predictive behavior.

\section{Machine Learning Models for Animal Behavior}

ML models grant a hypothesis-free way of creating models in very complex and large amounts of data. This characteristic of ML can prove to be very beneficial for modeling the complex social and behavioral iterations of animals. Currently, due to the advancements in technology, a scientist can capture more considerable amounts of data from monitoring animals that includes the animal's movements, social interactions, vocalizations, and even their physiological responses to outside stimulants. Classical statistics have proven to be inadequate in modeling and predicting the behavior in animals. An ML model can significantly improve the performance and predictive accuracy of these behaviors in an individual, group, or species level. Both traditional statistics and ML goals are to produce a mathematical model to show why these behaviors persist. There are considerable similarities and differences between these two methods. Statistics focus on inference on the data while ML looks to predict patterns in the data. ML also can use a hypothesis-free approach to modeling while traditional statistics relies on a hypothesis which can either be accepted or rejected based on the consistency with the observed data [11], [12].

There have been several successful applications of $\mathrm{ML}$ in animal behavior modeling. The use of GPS devices and video data to monitor the movement patterns of an individual. These devices can quickly produce an excessive amount of data every hour, which can then be used to classify the animals' activities and even predict where the animal will be or do in the future. This data can be used in an ML model to determine an individual's decision rules for migration and even measure how much time it spends on an activity. Vocalization is another area where ML has proven useful that can be used to predict the population size of a group or species and the interaction within the group or species. ML has even seen success with monitoring animal wellbeing when combined with notes and observations [11], [12].

There are two primary forms of ML, unsupervised and supervised learning. Unsupervised learning looks at the discovery of the data's structure in unlabeled data-sets. The discovery of structures in the data can be found by using visualization techniques. Supervised learning tends to be more closely related to traditional statistics in that it will find the relationships between variables and 
the results. Unlike statistics, an ML learns how to map the relationships between data and outcomes, which is where traditional statistics differs as it uses a predefined model structure first. The most significant advantage of using an ML to model animal behavior is that they can manage the nonlinear nature of the social behavior of animals and will be able to fit the data to the model. There is one downfall in that there is a small possibility to overfit or underfit the model or to model in noise that is usually present in data-set from the wild [11], [12].

\section{Tyrannosaurs Rex Background}

The T-Rex is arguably the most famous dinosaur that has ever lived. The TRex is one of the most studied dinosaurs and has appeared in many studies describing its behavior based off of the multiple fossils, techniques, simulations, and research. The T-Rex was a member of the tyrannosauroid family that is characterized by the smaller arm length and the possession of only two fingers. The majority of all the T-Rex fossils are on the western side of North America, which is where the majority of scientist believe the T-Rex originated from [13]. T-Rex Physical Characteristic:

The largest size of a T-Rex came to 4 meters tall and 12.3 meters long and weighted upwards to 9501 kilograms but averagely weighed around 6000-8000 kg. The T-Rex possessed strong legs which could push the dinosaur upwards of 17 to 40 kilometers per hour. It had a mighty tail that aided in the balancing of its colossal head which could get up to 1.5 meters long [14], [13]. The T-Rex bite force has been estimated to be close to 57,000 Newtons or roughly 12,814 poundsforce. Accompany this bite force, was a set of serrated teeth about $30 \mathrm{~cm}$ long. The front teeth specialized in gripping and pulling its live prey. The side teeth would be for ripping flesh from prey. Also, its back teeth designed for dicing up meat and pushing it back into its throat. The teeth were not sharp or dagger-like but more dull and broader than average hunters which allowed for the teeth to withstand the great force of its biting power and the struggling of prey. Its teeth also had deep roots to survive battles with prey [13], [14]. The T-rex's eyes were suited well for finding prey, and it has a very good olfactory lobe which would allow for a very heightened sense of smell. The T-Rex possessed a set of sturdy and strong legs which would allow for it to run faster than its prey, and its small forelimb would not hinder it from hunting as the risk of getting them damaged were slight, but they were still extremely powerful and could aid in combat. Most of the evidence that the T-Rex was a hunter comes from the dinosaurs that survived the attacks from them [4].

\section{T-Rex Diet and Hunting Behavior Patterns}

The T-Rex can is a carnivore in that most of his diet, energy, and nutrients come from eating other dinosaurs and meat sources. The T-Rex could have eaten upwards of 267 kilograms of meat in a single bite. Some researchers state that the T-Rex was merely an obligatory scavenger, while others scientist has stated that it was an obligatory hunter, but the more common approach is the middle ground with the T-Res doing a bit of both much like a lion today. There is even evidence that T-Rex even ate each other, especially the younger T-Rexes that have not reached maturity [15], [4],[16]. 


\section{Caloric Needs:}

In the animal kingdom, there are very few animals that are either obligate hunters or scavengers. This principle will also apply to the dinosaurs. It is tough to find enough food without wasting large amounts of energy in the process. The animal's movements must be extremely energy efficient and would not possess very massive muscles to quick running as their prey has no defenses at all. Even animals that regularly hunt like wolves and lions are known to scavenge dead animals from time to time [5]. So, the larger the size of the animal, the higher its daily caloric intake will be, the less likely it will be a pure scavenger. The T-Rex is an apex predator, but some may debate that it was purely an obligate scavenger or the opposite an obligate hunter. However, due to its size and daily caloric needs, many believe that the T-Rex was an opportunistic carnivore engaging in mainly active hunts as well as opportunistic scavenging when it could [17]. This behavior is due to the small amount of energy that the T-Rex would gain from pure scavenging, with as little as $500 \mathrm{~kg}$ of food from one theropod would not meet the requirements to sustain the massive T-Rex enough to solely rely on scavenging as its primary source of energy [18].

Teeth:

The teeth of animals can tell us a great deal about their eating habits and what they eat. The most common difference is between herbivores and carnivores. Herbivores have wide flat teeth for chewing vegetation and plant matter. Carnivores possess sharp pointed teeth to grip prey and to cut up flesh. So, the sharper and deeper the roots of the teeth, the more likely it will be a hunter over a scavenger. The T-Rex had roughly 50 teeth which were close to 8 inches in length. The teeth were both sharp and durable to cut through meat and crush bones. Another fact that if a T-Rex lost a tooth, the T-Rex could grow it back in less than two years [13].

\section{Size and Strength of Animals:}

The size of an animal does not mean that it is a hunter or scavenger per-say. The biggest animals are usually herbivores, like elephants or giraffes. However, when it comes to hunters, size makes the difference. The bigger and stronger the animal is, the easier it is to catch larger prey and to rely on hunting as the primary source of nutrition. The size of the dinosaur does not mean that it will not resort to scavenging, but this will not be the primary source of energy for the more predominately hunting inclined animals. Like lions, hyenas are mainly known as scavengers but have been seen killing their prey, much like lions, just to a much lesser extent [5]. The T-Rex was one of the largest dinosaurs that ever walked the earth and was one of the biggest carnivores that dominated in its time. During the T-Rex reign, no known carnivore that could come close to it which makes it the apex predator of its time. Due to the T-Rex's size and strength, it could easily take down another dinosaur during a hunt. Research has shown that the T-Rex was faster than its usual prey which would allow for it to chase down his prey much like a lion of today [4], [14]. Combined with its caloric needs and its bio-mechanical build, this would allow for some to speculate that the T-Rex was primarily a hunter first and an opportunistic scavenger second. 


\section{Dataset}

The dataset for the model will look at many of the physical characteristic of the many hunter and scavenger animals to classify what type of hunter the T-Rex may have been. The physical characteristic is; teeth size, biting power, estimate running speed, the speed of primary prey, size, size of primary prey, daily caloric needs, eyesight type, and sense of smell. These characteristics were all chosen as the most hunter will have a very high level of each of the characteristics compared with the characteristics of an obligatory scavenger. The dataset only has carnivores' physical characteristics as herbivores would add noise to the data set and are outside of this pilot study as well as the studies goal of classification of hunting behavior patterns.

The input data for the model will consist of several variables that every animal share. The first variable will be the length of the teeth of the animal, which plays a vital role in determining feeding behavior [25]. The total estimated bite force of the animal will also be a variable as this can limit the type of prey the animal can attack [26]. The weight or size of the animal, as well as its primary prey, will also be a variable as this is a primary factor in determining the total number of calories needed per day [5], [18]. The length of the animal is also significant as larger animals tend to be apex predators over a smaller one. The estimated maximum speed of both the predator and prey can show if the predator can engage in active predation [27]. Both eyesight and sense of smell will also be useful as these senses are keen for active predation. These input data variables should play a significant roll in helping model the actual behavior of each animal. Some of the input variables may be discarded in further studies as they may not influence the outcome or maybe too general to every species.

There were many species of animals used in the dataset. There are several theropods included in the study consisting of a T-Rex, Utahraptor, Allosaurus, and the Spinosaurus which are the more well-known theropods due to cinema and pop culture. The other animals are more common living animals that consist of the only living 'dinosaur' the crocodile, the lion, the bear, the tiger, the hyena, the jaguar, the cheetah, and the Komodo dragon. This selection of scavengers and hunters with different niche hunting techniques only strengthens the model's classification ability. The dataset is small as the data is challenging to collect as sources are few and far between.

The testing data set will come from both modern and extinct animals. The use of modern animals will help give higher accuracy as their behaviors are known and well documented a ground truth as well, in a sense. The following table will show the common characteristic of a male animal found in the wild.

\begin{tabular}{|c|c|c|c|c|c|c|c|c|c|c|c|c|}
\hline Name & TeethLength & Weight & Length & Height & Speed & Calorie Intake & Bite Force & Prey Speed & PreySize & EyeSight & Smell & Class \\
\hline T-Rex & 12 & 15432 & 40 & 20 & 33 & 40000 & 12800 & 20 & 19841 & 0 & & OPrimary Hunter \\
\hline Crocodile & 4 & 2400 & 23 & 1.6 & 8 & 2500 & 3700 & 30 & 881 & 0 & & OPrimary Hunter \\
\hline Lion & 2.7 & 416 & 9.8 & 3.9 & 50 & 7236 & 650 & 35 & 1300 & 0 & & OPrimary Hunter \\
\hline Bear & 3.6 & 600 & 7 & 3.35 & 40 & 20000 & 975 & 0 & 0 & 0 & & 0 Primary Scavenger \\
\hline Tiger & 3 & 260 & 12 & 3 & 40 & 7236 & 1050 & 37 & 160 & 0 & & OPrimary Hunter \\
\hline Hyena & 0.27 & 160 & 5 & 2 & 37 & 5000 & 1100 & 20 & 40 & 0 & & 0 Primary Scavenger \\
\hline Jaguar & 2 & 220 & 5.5 & 2.5 & 40 & 5000 & 1350 & 15 & 300 & 0 & & OPrimary Hunter \\
\hline Cheetah & 1.5 & 154 & 4.9 & 2.9 & 70 & 2200 & 475 & 56 & 185 & 0 & & OPrimary Hunter \\
\hline Komodo Dragon & 0.4 & 150 & 8.5 & 1 & 13 & 1994 & 240 & 24 & 110 & 0 & & 0 Primary Scavenger \\
\hline
\end{tabular}

Table 1: Testing Data for different animals. 
This data will give an excellent example of how the model can classify the eating behavior and can also help provide better proof that this model has the potential for use and further study.

\section{Methodology}

The primary language is python because this language is trendy for ML and possess many libraries to aid in data preprocessing, model building, training, visualization, and testing of ML models. The primary ML library is scikit-learn as this gives the primary data processing steps as well as many of the models for testing. This library increases the speed for training, testing, and analytically analysis. Scikit-learn is preprocessing can be useful in deep learning models in the future so less boilerplate code.

There will be a few algorithms used in this study to aid in the classification of animal behavioral patterns. The algorithms are; $\mathrm{K}$ nearest neighbor, logistic regression, support vector modeling (SVM) model, latent Dirichlet allocation, Naive Bayes, and a decision tree algorithm. Comparing the results of these three models will tell which one the more accurate predictor and which models are should continue as a baseline model for comparison to a more complex model like a neural net or deep learning implementation.

KNN is a nonparametric method that is well suited for classification problems. The input is the $\mathrm{k}$ closest training example in a feature space, while the output is base on its usage. For classification, the output will be a class membership. The determination of the classification is by the majority vote of its immediate neighbors. If $\mathrm{k}=1$, then the object is assigned the class of that single nearest neighbor [19].

Logistic regression is a statistical model that is useful for a binary dependent variable by estimating the parameters of the logistic model. The two possible values for the dependent variable can either be 0 or 1 . Logistic regression can also be generalized to more descriptive levels of dependent variables by having categorical outputs by using ordinal logistic regression [20].

SVM is a supervised learning model that works well for regression and classification problems. SVM works by receiving a set of data that is marked to belong to one or another category. The SVM builds the model that will assign new examples to one of the categories. SVM is useful for unsupervised learning, where the algorithm will try to find the natural groups or clusters be data points and map new data to one of these points [21].

LDA is a generative statistical model where observations can be explained by unobserved groups that can show the relationship between why some data is similar to each other [22].

A decision tree is a support tool that produces a graph of a decision and any possible outcomes which includes even slight chances of any outcome. The representation of the outcome of a test where each leaf node represents a class label and each path to a new leaf is a classification rule [23].

Naive Bayes is a simple probabilistic classifier that can be used to construct classifiers that assign class labels to a problem instance. Vectors of a feature can represent the class values where each label come from a finite set of classes. Naive Bayes assumes that the value of a feature is independent of any of the feature given a class variable [24]. 
The comparison of each of the models will look at how well each model perform at prediction. The evaluation of each algorithm will consist of a confusion matrix and accuracy scores. The models will be scored based on the accuracy of the predictions and classification of different animals. The results will be analyzed, and the best models will be a baseline for further studies.

Test Case:

The T-Rex is chosen as the test case as the dinosaur is as an opportunistic scavenger but a primary hunter. The T-Rex would be primarily classified as a hunter as the daily caloric intake needs would not allow for the T-Rex rely on obligatory scavenging. The T-Rex biting power is suggested to be the most potent land-based biting power that has ever lived. The strength of the bite force is indicative of a hunter in that scavengers do not have such a great need for biting power as their prey have no defenses. The T-Rex teeth were designed to survive struggles of a battle, cut through flesh, and to crack bone easily. The estimated speed of the T-Rex is estimated to be faster than its primary prey. The T-Rex possessed excellent eyesight and had binocular vision which would help judge for depth when hunting and attacking prey. Based on the structure of the T-Rex's head, it had an excellent sense of smell and could track animals across long distances [4],[14], [13], [17].

\section{Results}

The result was helpful in that it shows which model to use for further studies. The models that will continue for further analysis, as well as the baseline for further model development, will be the logistic regression model, LDA model, Decision Tree model, and the SVM as they all scored high in accuracy and had no miss-classifications on the confusion matrix. Two models that failed is the KNN and the Naive Bayes as their accuracy were both $66 \%$ and misplaced three scavengers for the KNN and three hunters for NB (please see Appendix: Classification Report).

The use of the T-Rex, along with other animals that are currently living today allows for a better idea about how these models can be useful in the classification of animal behaviors. Each model's results look promising, especially the results on the T-Rex. The classification of the T-Rex as a primary hunter for every single model does show promise which matches with the literature [17]. However, these results can be misleading in turn. Most of the training data come from living animals and only a hand full of theropods. This data can skew the results as the most massive teeth would naturally be a hunter. However, the use of living animals for behavior modeling of theropods is not a new technique and is commonly applied today. The lack of multiple types of theropods may also skew the results for this study as the models would learn more based on living animals rather than the dinosaurs, which are the target of the study.

\section{Conclusions}

By looking at the physical characteristic of an animal, a mathematical model can be generated to help in the classification of behavioral patterns in several different areas ranging from a territory, movement and migration patterns, social 
interactions, and eating behaviors. The use of conventional machine learning models for a pilot study shows proof that ML can be useful for animal-behavior prediction and classification by using the animal's physical characteristic and biomechanics that drive the animals' interaction between its environment as well as its prey. This pilot study was a simple study to start research into the use of ML and artificial intelligence in the field of paleontology. Further research needs to do to improve on this foundation set out in this paper. The results from the ML models that were good overall with only 2 out of 6 of the models making a mistake with only a miss-classification of 3 animals. However, all the models did successfully classify the T-Rex as a hunter over a scavenger which is what the expected outcome was.

\section{Future Work}

This paper is a pilot study to further the use and to create a stepping stone for the usefulness of ML techniques in paleontology and animal studies. ML is gaining popularity in many fields as a powerful tool and can quickly solve many complex problems. By using the results from this study, further development of a more accurate and useful classification method for objective classification of dinosaurs can be built to aid in the further advancement of paleontologist and applications of ML. The next steps would be to look at more features like habitats, pack hunting, more characteristic of primary prey, looking at modern animal patterns, and even other behavioral patterns not in this pilot study that affect hunting behaviors.

The most significant step would be to collect a more extensive dataset consisting of only theropods rather than any living animals. This new dataset would then be the training data for a deep learning model which should be far more accurate over the simpler ML algorithms. Due to the rarity of full skeletal finds of theropods, some advance techniques of data augmentation will have to be implemented.

\section{References:}

Horner, J. Dinosaur behavior and growth. The Paleontological Society Special Publications; 1992, 6, 135-135.

Chiappe, LM. A Field Trip to the Mesozoic. PLOS Biology; 2003 1(2): e40.

Tanke, D. Currie, P. Head-Biting Behavior In Theropod Dinosaurs: Paleopathological Evidence. gaia: Okologische Perspektiven in Natur-, Geistes- Und Wirtschaftswissenschaften; 2000, 15, 167-183.

Holtz, T.R. A Critical Reappraisal of the Obligate Scavenging Hypothesis for Tyrannosaurus rex and Other Tyrant Dinosaurs. in Larson, P. and Carpenter, K. (eds) Tyrannosaurus rex: The Tyrant King. Bloomington: Indiana University Press; 2008.

DeVault, T. Rhodes, O. Shivik, J. Scavenging by vertebrates: behavioral, ecological, and evolutionary perspectives on an important energy transfer pathway in terrestrial ecosystems. USDA National Wildlife Research Center - Staff Publications; 2003, 269.

Sherman, P. W., \& Seeley, T. D. Animal behaviour. Retrieved July 23, 2018, from https://www.britannica.com/science/animal-behavior/History-and-basic-concepts; 2017.

McGreevy, P. Boakes, R. Carrots and Sticks; Principles of Animal Training. Darlington Press; 2011. Pg xi-23.

Immelmann, K. (). Aims, Methods, and Areas of Ethology. Introduction to Ethology; 1980, 1-9.

Boakes, R. From Darwin to behaviourism: Psychology and the minds of animals. Cambridge: Cambridge University Press; 1988. 
Anderson, D. Perona, P. Toward a Science of Computational Ethology, Neuron; 2014 Volume 84, Issue 1, Pages 18-31.

Valletta, J. J., Torney, C., Kings, M., Thornton, A., \& Madden, J. Applications of machine learning in animal behaviour studies. Animal Behaviour; 2017,124, 203-220.

Schank J., Joshi S., May C., Tran J.T., Bish R. A Multi-Modeling Approach to the Study of Animal Behavior. In: Minai A.A., Braha D., Bar-Yam Y. (eds) Unifying Themes in Complex Systems. Springer, Berlin, Heidelberg; 2011.

Castro, J. Tyrannosaurus Rex: Facts About T. Rex, King of the Dinosaurs. Retrieved July 19, 2018, from https://www.livescience.com/23868-tyrannosaurus-rex-facts.html; 2017.

Hutchinson, JR. Bates, KT. Molnar, J. Allen, V. Makovicky, PJ. A computational Analysis of Limb and Body Dimensions in Tyrannosaurus Rex with Implications for Locomotion, Ontogeny, and Growth. PLoS One; 2011, vol 610

Longrich, N., Horner, J., Erickson, G., \& Currie, P. Cannibalism in Tyrannosaurus rex. PloSOne; 2010. 5(10): e13419

Horner, J., Goodwin, M., \& Myhrvold, N. Dinosaur Census Reveals Abundant Tyrannosaurus and Rare Ontogenetic Stages in the Upper Cretaceous Hell Creek Formation (Maastrichtian), Montana, USA PLoS ONE; 2011, 6 (2)

Hutchinson, John. Tyrannosaurus rex: predator or media hype?. What's in John's Freezer? Retrieved August 26, 2013.

Kane, A. Healy, K. Ruxton, G. Jackson, A. "Body Size as a Driver of Scavenging in Theropod Dinosaurs." The American Naturalist; 2016, 187(6).

Altman, N. S. An introduction to kernel and nearest-neighbor nonparametric regression. The American Statistician; 1992, 46(3): 175-185.

Walker, SH. Duncan, DB. Estimation of the probability of an event as a function of several independent variables. Biometrika; 1967. 54: 167-178.

Cortes, Corinna; Vapnik, Vladimir N. Support-vector networks. Machine Learning; 1995, 20 (3): 273297.

Blei, David M.; Ng, Andrew Y.; Jordan, Michael. Lafferty, John, ed. Latent Dirichlet Allocation. Journal of Machine Learning Research; 2003. 3 (4-5): pp. 993-1022

Quinlan, J. R. Simplifying decision trees. International Journal of Man-Machine Studies; 1987,27 (3): 221.

Russell, Stuart; Norvig, Peter. Artificial Intelligence: A Modern Approach (2nd ed.). Prentice Hall; 2003.

Rayfield, E. J. (). Cranial mechanics and feeding in Tyrannosaurus rex. Proceedings of the Royal Society of London. Series B: Biological Sciences; 2004, 271(1547), 1451-1459

Meers, M. B. Maximum Bite Force and Prey Size of Tyrannosaurus rex and Their Relationships to the Inference of Feeding Behavior. Historical Biology; 2002, 16(1), 1-12.

Hutchinson, J. R. Biomechanical modelling and sensitivity analysis of bipedal running ability. II. Extinct taxa.J. Morphol; 2004. 262, 441-461.

\section{Appendix: Classification Report}




\begin{tabular}{|l|l|l|l|l|}
\hline & precision & recall & f1-score & support \\
\hline 0 & 1.00 & 1.00 & 1.00 & 6 \\
\hline 1 & 1.00 & 1.00 & 1.00 & 3 \\
\hline avg / total & 1.00 & 1.00 & 1.00 & 9 \\
\hline
\end{tabular}

LDA

\begin{tabular}{|l|l|l|l|l|}
\hline & precision & recall & f1-score & support \\
\hline 0 & 1.00 & 1.00 & 1.00 & 6 \\
\hline 1 & 1.00 & 1.00 & 1.00 & 3 \\
\hline avg / total & 1.00 & 1.00 & 1.00 & 9 \\
\hline
\end{tabular}

KNN

\begin{tabular}{|l|l|l|l|l|}
\hline & precision & recall & f1-score & support \\
\hline 0 & 1.00 & 0.67 & 0.80 & 9 \\
\hline 1 & 0.00 & 0.00 & 0.00 & 0 \\
\hline avg / total & 1.00 & 0.67 & 0.80 & 9 \\
\hline
\end{tabular}

Decision Tree

\begin{tabular}{|l|l|l|l|l|}
\hline & precision & recall & f1-score & support \\
\hline 0 & 1.00 & 1.00 & 1.00 & 6 \\
\hline 1 & 1.00 & 1.00 & 1.00 & 3 \\
\hline avg / total & 1.00 & 1.00 & 1.00 & 9 \\
\hline
\end{tabular}

Naive Bayes

\begin{tabular}{|l|l|l|l|l|}
\hline & precision & recall & f1-score & support \\
\hline 0 & 0.50 & 1.00 & 0.67 & 3 \\
\hline 1 & 1.00 & 0.50 & 0.67 & 6 \\
\hline avg / total & 0.83 & 0.67 & 0.67 & 9 \\
\hline
\end{tabular}

SVM

\begin{tabular}{|l|l|l|l|l|}
\hline & precision & recall & f1-score & support \\
\hline 0 & 1.00 & 1.00 & 1.00 & 6 \\
\hline 1 & 1.00 & 1.00 & 1.00 & 3 \\
\hline avg / total & 1.00 & 1.00 & 1.00 & 9 \\
\hline
\end{tabular}

